\title{
Chiral Surface Characterisation and Reactivity Toward H-D Exchange of a Curved Platinum Crystal
}

\author{
Tycho Roorda ${ }^{1} \cdot$ Sabine V. Auras ${ }^{2} \cdot$ Ludo B. F. Juurlink $^{2}$ (i)
}

Published online: 11 October 2020

(c) The Author(s) 2020

\begin{abstract}
Understanding heterogeneous catalysis at the atomic level requires detailed knowledge of the reactivity of different surface sites toward specific bond breaking and bond making events. We illustrate a new method in such investigations. We use a macroscopically curved Pt single crystal containing a large variation in density of highly kinked steps of two different chiralities. Scanning tunneling microscopy maps the entire range of surface structures present on the $31^{\circ}$ section surrounding the $\mathrm{Pt}(111)$ apex. Whereas most of the surface shows the expected characteristic arrays of parallel steps, hexagonally-shaped, single-atom deep pits remain after cleaning procedures near the apex. Their orientation is indicative of the different chiralities present on the two sides of the crystal's apex. These unintended defects locally raise the surface defect concentration, but are of little consequence to subsequent reactivity measurements for $\mathrm{D}_{2}$ dissociation and $\mathrm{H}-\mathrm{D}$ exchange as probed by supersonic molecular beam techniques. We quantify absolute elementary dissociation and relative isotopic exchange rates across the surface with high spatial resolution. At low incident energies, elementary dissociation of the homonuclear isotoplogues is dominated by the kinked steps. $\mathrm{H}-\mathrm{D}$ exchange kinetics depend also mostly linearly on step density. The changing ratio of $\mathrm{D}_{2}$ dissociation to H-D formation, however, suggests that anisotropic diffusion of H(D) atoms is of influence to the measured HD production rate.
\end{abstract}

Keywords Platinum $\cdot$ Hydrogen $\cdot$ Single crystal $\cdot$ Curved $\cdot$ Catalysis $\cdot$ Molecular beam $\cdot$ H-D exchange

$\begin{array}{ll}\text { Abbreviations } \\ \text { Pt } & \text { Platinum } \\ \text { Ar } & \text { Argon } \\ \text { STM } & \text { Scanning tunnelling microscopy } \\ \text { LEED } & \text { Low-energy electron diffraction } \\ \text { TW } & \text { Terrace width }\end{array}$

T. Roorda and S. V. Auras are equally contributing authors.

Electronic supplementary material The online version of this article (doi:https://doi.org/10.1007/s11244-020-01371-3) contains supplementary material, which is available to authorized users.

Ludo B. F. Juurlink

1.juurlink@chem.leidenuniv.nl

1 Leiden Institute of Physics, Leiden University, PO Box 9504, 2300 RA Leiden, The Netherlands

2 Leiden Institute of Chemistry, Leiden University, PO Box 9502, 2300 RA Leiden, The Netherlands

\section{Introduction}

Many studies in catalytic surface science make use of high symmetry, low Miller index planes of metal single crystals [1]. While they are the simplest types of surfaces to describe theoretically, experimentally a perfect, defect-free flat surface as described by theory, cannot be produced. Furthermore, real life chemical reactions at surfaces, such as they occur in heterogeneous catalysis or environmental chemistry, occur on surfaces featuring a multitude of sites [2-5]. Undercoordinated sites on surfaces, such as steps, kinks, edges and corners, are often found to be more reactive towards dissociation of reactant molecules and may be responsible for the dominant part of a catalyst's activity.

One way of studying more realistic surfaces involves the use of nanocrystals. Norbert Kruse and others explored this field already in the 1980's making use of techniques such as Field Ion Microscopy (FIM) in combination with pulsed field desorption mass spectrometry [6, 7]. The atom-probe technique is extremely powerful, visualizing multiple crystal planes that may contribute to the catalytic process at once. Kruse and colleagues showed, for example, that surface 
chemical reactions, oscillations in reactivity, and restructuring of facets can be studied under a rather wide range of experimental conditions. For example, nanocrystalline $\mathrm{Pt}$ field emitter tips were used to elucidate different catalytic rates and processes for $\mathrm{NO}$ and $\mathrm{NO}_{2}$ reduction on Pt facets [8-15]. There, tips were specifically shaped to expose facets of interest. Kruse continues to study different catalytic processes and different metallic and alloy catalysts.

Another approach to studying the influence of surface heterogeneity to surface chemistry and catalysis employs flat single crystals of planes vicinal to low-Miller index directions. This is particularly useful in studies that require a macroscopic flat area of a single surface structure, e.g. one with a fixed number density of particular types of steps and/ or kinks. Molecular beams, often used to study the dynamics of (activated) adsorption and overall reactions with control over the kinetic energy of the reactant(s), require such larger flat areas characterized by a single surface unit cell [16]. The lower symmetry of these unit cells make them more challenging to study with accurate theoretical methods. Nonetheless, the dynamics of molecule-surface collisions with vicinal surfaces have been subject of several theoretical and experimental studies, some even including rovibrational state-selection of the incident molecule [17-23].

The approach using vicinal surfaces requires that the same reaction is studied on several different surface structures to extract site-specific reactivities [24-28]. Comparing reactivities of single crystals with varying number densities of particular types of terraces, steps, or kinks may yield direct and quantifiable evidence for the influence of each particular type of site on the reaction. Experimentally, this is tedious, though. Subtle differences may be difficult to identify or hidden between variations in, e.g., contamination levels or sensitivity to surface restructuring of the various crystal surfaces. Deliberately introducing various levels of sputter defects to a nearly perfect single crystal surface presents a shortcut to the approach $[29,30]$. It unfortunately grants poor control over the types of defects that are introduced.

Curved surfaces of single crystals have recently been reinvented as a powerful tool to elucidate the role of specific surface sites in catalytic reactions [31-33]. While in the past many different types of curved surfaces have been used, in the last decade the predominantly used shapes are a small section of a cylinder and a dome [31]. Most commonly, the crystallographic orientation is chosen to exhibit a low-Miller index surface at the apex. The macroscopic curvature of the crystal results predominantly from monoatomic steps occurring with increasing frequency when moving away from the apex. Depending on the azimuthal angle, these steps may be atomically straight or kinked. This curved crystal approach to studying the influence of surface structure in catalysis strongly resembles the nanocrystal approach employed by Kruse - both use a single sample that contains a range of surface structures.
The macroscopic size of these new curved crystals, however, allows for much smoother variation of surface structure. They often exhibit vicinal surfaces with a well-defined, continuous range of step densities. Although they do not reflect the shape of actual catalytic particles, the gradual variation in surface densities of various types of active sites make them ideal to unravelling individual contributions from such sites to overall reactivity.

We have recently determined site-specific reaction parameters of $\mathrm{D}_{2}$ dissociation on stepped and kinked Pt(111) surfaces $[32,34]$. We have used two different curved Pt single crystals with (111) at the apex. One crystal provided close packed steps of $\{001\}$ and $\{110\}$ orientation (A- and B-type steps respectively) on either side of the apex. The second crystal had kinked steps consisting of short segments of A- and B-type interspersed by inner and outer kinks [34]. Due to their orientation, the latter should nominally be fully kinked, however, we observed a partial reconstruction of the kinked step edges into slightly longer A- and B-type segments with a reduced kink density. Specific Pt surfaces are very sensitive to the cleaning treatment and may be prone to reconstructions and faceting $[35,36]$. On a similar cylindrical Pt single crystal with a [001] axial direction, Imbihl et al. observed reconstructions and step doubling on parts of the curved surface [37].

Considering the possible instabilities on vicinal Pt surfaces, it is important to fully and in detail characterise the surface of a curved crystal. This is especially important when trying to unravel the influence of specific surface sites, such as kinked steps, that increase the surface free energy. Here, we present an investigation of the surface structures along the curvature of a $c-\operatorname{Pt}(111)[11 \overline{2}]-31^{\circ}$ crystal, i.e. a $31^{\circ}$ curved section of a cylinder with (111) at the apex and the rotational axis pointing in the [112] direction [31]. Determining the densities of intentional and non-intentional (residual) defects allows us to critically consider what areas of the curved surface can be used in evaluating absolute reactivities of specific surface sites. Notably, for this particular crystal, residual hexagonally shaped defects also indicate the handedness of the kinked step causing the macroscopic curvature. We subsequently show that we may extrapolate the previously reported reactivity of $\mathrm{D}_{2}$ dissociation towards an otherwise unattainable perfect, defect-free $\mathrm{Pt}(111)$ surface. We finally show that the $\mathrm{H}-\mathrm{D}$ exchange rate between $\mathrm{H}_{2}$ and $\mathrm{D}_{2}$, which Norbert Kruse developed as a technique to determine the surface area of metal catalysts [38], mostly tracks the elementary dissociation of the reactants.

\section{Experimental}

The $8 \times 7 \mathrm{~mm}$ Pt curved single crystal was prepared by spark erosion and sanding at Surface Preparation Laboratories (Zaandam, The Netherlands). The crystal shape is 
depicted in Fig. 1a. It curves $15.5^{\circ}$ to both sides from the (111) plane at the apex, with a $15 \mathrm{~mm}$ radius of curvature around the $[11 \overline{2}]$ axis. Towards the sides of the crystals, the (111) terraces become shorter as the step density increases linearly towards the edge (Fig. 1b). Concomitantly, the terrace width decreases with $1 / \mathrm{x}$, where $\mathrm{x}$ is the distance from the apex. Due to the direction of curvature, the steps causing macroscopic curvature are in principle the most highly kinked steps. They are illustrated by purple spheres in Fig. 1c. The kinked steps consist of alternating $\{001\}$-oriented and $\{110\}$-oriented microfacets, denoted as A-type (blue spheres) and B-type (red spheres) respectively. Due to the reduced symmetry of the kinked steps, surfaces with opposite chirality are formed on the two sides of the crystal. The notation of chirality for kinked steps on $f c c(111)$ terraces, as described by McFadden et al. [39], is obtained by ordering the microfacets around an inner kink by their atomic density. Going from the $\{111\}$ terrace to the $\{100\}$ segment to the $\{110\}$ segment, we observe a counterclockwise rotation, denoted by $(S)$ in the left depiction of Fig. 1c, and vice versa for $(R)$ chirality.

With increasing distance from the apex (x), the angle of curvature $\alpha$ increases as defined by the radius of curvature $\mathrm{R}$. At the same time, $\alpha$ determines the angle between the (111) normal and the vicinal surface normal. Therefore, on a microscopic level, $\alpha$ links the average terrace width (TW) of the stepped surfaces, to the height between atomic layers, $\mathrm{h}=0.226 \mathrm{~nm}$. Figure $1 \mathrm{~d}$ demonstrates the relationships between macroscopic (x, R) and microscopic (TW, h) parameters and $\alpha$. We obtain the following relation to determine TW along the curved crystal:

$T W=\frac{h}{\tan (\alpha)}=\frac{h}{\tan \left(\sin ^{-1}(x / R)\right)}=\frac{h \cdot(R-\epsilon)}{x}$

The size and shape of this crystal thus, in principle, encompasses (111) terrace widths ranging from very large at the apex to approximately 3 atoms wide at the edges of the crystal. However, the polished section of the curved surface
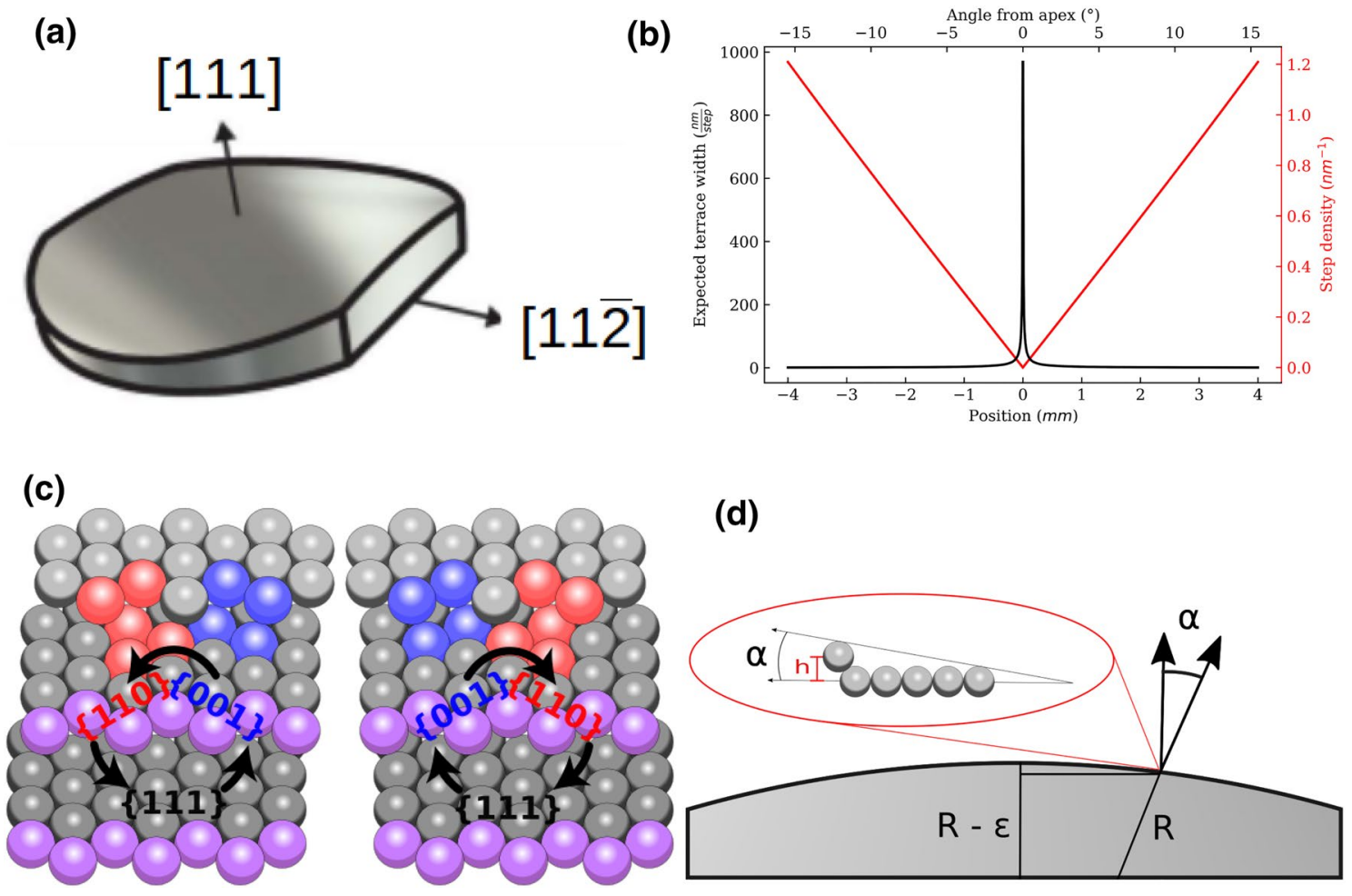

(d)

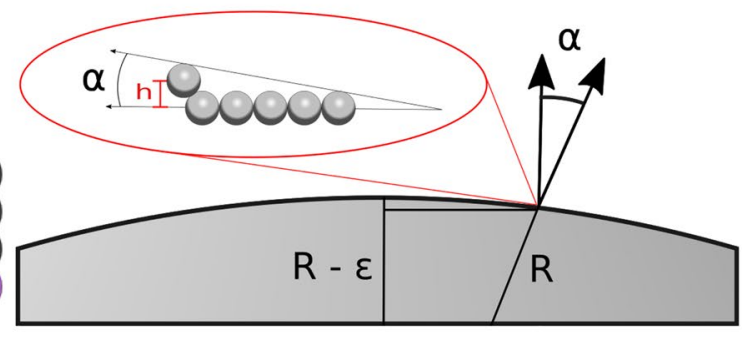

Fig. 1 a Schematic drawing of our platinum crystal curving perpendicular to the $(11 \overline{2})$ plane. Adapted from van Lent et al. [32]. b Expected terrace widths and step densities along the curvature of the crystal, calculated for monoatomic height steps, represented in distance from the apex and angle. The matching results from STM imaging are shown in the supplementary information. c Crystal structure of $f c c\{432\}$ planes showing the kinked step consisting of short segments of A-type (blue) and B-type (red) steps. The chirality in the left and right images, identified as $(S)$ and $(R)$ chirality respectively, represent the surfaces on opposing sides of the curvature. d Depiction of a side view of the crystal with a zoom-in of a single terrace with adjacent step atom to indicate how the terrace width is calculated. The terrace width, TW, is determined by the height of a step, $h=0.226 \mathrm{~nm}$, and the angle $\alpha$ which represents the angle between the (111) terrace normal and the stepped surface normal, as well as the angle between the apex and the position along the curvature with position $\mathrm{x}$ and radius $\mathrm{R}$ in the equivalent triangle 
tapers toward the outer edges and we can not use the full width of $7 \mathrm{~mm}$ along the entire surface. As discussed below, we mostly use the available area ranging to approximately 5 atom wide terraces to unravel site-specific reactivities.

The surface of our curved crystal is cleaned and analyzed initially using a commercial UHV system with a variable temperature scanning tunning microscope (VT-STM, Omicron). It has been altered in-house and contains, a.o., optics for Auger Electron Spectroscopy (AES) and Low Energy Electron Diffraction (LEED). We clean the crystal's surface by repeated cycles of argon ion sputtering, oxygen or hydrogen annealing and vacuum annealing. The sample can be heated radiatively up to $\sim 575 \mathrm{~K}$ by a homemade $\mathrm{W}$ filament, higher temperatures are reached by electron bombardment when a positive potential is applied to the sample. A typical cleaning cycle consists of $\mathrm{Ar}^{+}$sputtering at room temperature, $0.52 \mathrm{keV}$ and $7 \mu \mathrm{A}$ for a duration of 10 minutes at $1.3 \times 10^{-5} \mathrm{mbar}$ followed by oxygen annealing at $3 \times 10^{-8}$ mbar and $\sim 900 \mathrm{~K}$ and finished with vacuum annealing at $1200 \mathrm{~K}$. Due to potential sensitivity of the vicinal surfaces to restructuring and faceting at $1200 \mathrm{~K}[35,36]$, the final cleaning cycle consists only of sputtering and annealing in vacuo at a reduced temperature. To reduce the formation of deep pits during sputtering, the surface temperature is raised during the last sputtering step, to facilitate adequate subsequent annealing. In the STM apparatus, the last cleaning cycle consisted of $\mathrm{Ar}^{+}$sputtering at an elevated temperature of $573 \mathrm{~K}$ (no electron bombardment) followed by vacuum annealing at $773 \mathrm{~K}$, each for 5 minutes. Annealing temperatures $>700 \mathrm{~K}$ ensure increased interlayer Pt atom mobility on the surface due to evaporation from the step edges [40]. We use the AES and LEED optics to verify the chemical purity and surface structure of the crystal surface. Results of the LEED study are shown in the supplementary information. For our analysis of intentional and non-intentional (residual) defects on the surface, the surface is imaged by STM along the curvature in a single line. Although our system in principle allows for cooling of the sample temperature, all of our STM imaging was performed with the crystal near room temperature. We have taken $\sim 1000$ images $(2$ direction scans per position). Of these, $\sim 150$ suitable images were selected for quantitative analysis. The apex can be easily located by where the surface steps change in direction. Distance travelled along the surface is regulated by counting clicks with a stepping motor.

To determine structure-dependent chemical reactivities, the crystal is mounted in the UHV chamber of a second apparatus, i.e. a home-built system that also contains multiple chambers for creating a supersonic molecular beam $[32,34]$. Here, a slightly modified cleaning procedure is applied, consisiting of repeated cycles of sputtering $\left(\mathrm{Ar}^{+}\right.$, $1 \mathrm{keV}, 1.3 \mu \mathrm{A}, 5 \mathrm{~min})$, annealing in $\mathrm{O}_{2}\left(3 \times 10^{-8} \mathrm{mbar}\right.$, $900 \mathrm{~K}, 3 \mathrm{~min})$ and annealing in vacuum $(1200 \mathrm{~K}, 3 \mathrm{~min})$.
The technical setup in the second apparatus made it possible to use electron bombardment during sputtering by applying a negative potential to the filament used for heating, allowing us to sputter at an elevated temperature of $900 \mathrm{~K}$, followed by annealing in vacuum $(900 \mathrm{~K})$. As a consequence, there are likely even less non-intentional defects during molecular beam experiments than during the STM study. We then impinge molecules from a supersonic molecular beam onto well-defined parts of the sample. The molecular beam is formed by supersonic expansion of gas mixtures with subsequent skimming of the gas plume and two stages of differential pumping prior to the beam entering the UHV chamber that holds the curved Pt sample. The beam's final skimmer is rectangular. It projects a $0.126 \mathrm{~mm}$ high and 6.0 $\mathrm{mm}$ wide beam along the (111) normal onto different parts of the curved crystal. The height of the beam limits convolution with the varying step density along the curved surface.

Initial dissociation probabilities for $\mathrm{D}_{2}, \mathrm{~S}_{0}\left(\mathrm{D}_{2}\right)$, are determined using the Kind-and-Wells method [41]. A $\mathrm{D}_{2}$ beam anti-seeded with $\mathrm{Ar}$ is used. $\mathrm{D}_{2}$ molecules impinge with an average kinetic energy of $9.3 \mathrm{meV}$ as determined by time-of-flight measurements using a quadrupole mass spectrometer (QMS) mounted on-axis in the UHV chamber of the system (UTI100c) with the crystal retracted from the beam, and the beam being chopped at high frequency into short pulses in the first differential pumping stage. For the dissociation probability measurements, the surface is kept at $\mathrm{T}_{\mathrm{s}}=155 \mathrm{~K}$. At this temperature, molecular hydrogen sticks via dissociative adsorption. Recombinative desorption is avoided by keeping the surface temperature far below the onset of desorption from $\mathrm{Pt}(111)$ and comparable vicinal surfaces [42-46].

For determining relative HD formation rates, a mixed beam with equivalent parts of $\mathrm{H}_{2}$ and $\mathrm{D}_{2}$ is used. Again, it is anti-seeded with Ar to achieve a similar kinetic energy as during $\mathrm{D}_{2}$ sticking. The crystal is kept at $500 \mathrm{~K}$, i.e. well above the recombinative desorption temperature of small surface concentrations of atomic $\mathrm{H}$ (D). This high temperature in combination with the limited flux of molecular $\mathrm{H}_{2}$ $\left(\mathrm{D}_{2}\right)$ ensures a low steady state atomic $\mathrm{H}(\mathrm{D})$ coverage during exposure to the beam. Relative HD formation rates are measured using a second QMS located in the UHV system (Pfeiffer, QMA200). We open a beam shutter in the second differential pumping stage to only allow a narrow part of the surface to be bombarded by the mixed $\mathrm{H}_{2}-\mathrm{D}_{2}$-Ar beam. We wait for a few seconds to obtain a stable current at $\mathrm{m} / \mathrm{e}=3$ as determined by the QMS prior to shutting the beam. Measurements of HD formation are taken for several non-overlapping parts on the surface by moving the crystal relative to the beam within the course of several minutes. Thereafter the crystal is cleaned and experiments are repeated using various orders and different sections along the crystal's curved surface. The QMS signal is corrected for the background 
current obtained at $\mathrm{m} / \mathrm{e}=3$ at the times intermittent to beam exposure. Relative reactivities are reported as the QMS current produced only resulting from HD formation by the crystal's local surface structure during initial exposure to the beam. As we have recently demonstrated that HD formation is more sensitive to structural changes than even LEED [47, 48], the consistent linear increase of HD formation with step density presented in Fig. 3 confirms that no step doubling or facetting is occuring.

\section{Results and Discussion}

\subsection{STM Analysis of Intentional and Non-intentional Defects}

We start by describing our general observations from the STM analysis. At the apex, atomically flat terraces as long as $1500 \mathrm{~nm}$ are observed along with areas showing heavy step bunching. On most larger terraces, single layered vacancy islands are found in between parallel steps. We attribute this to the relatively short annealing time at low temperature during the last step of our cleaning routine [40, 49]. Towards $\sim 100 \mu \mathrm{m}$ from the apex, the number of vacancy islands per area decreases and less step bunching is observed, however, step edges still meander slightly. As the step density increases, gradually the lines straighten out along the inherent step direction. Terrace widths are measured by drawing lines scans from step edge to step edge, neglecting vacancy islands as the end of a terrace.

Our general observations are illustrated in the STM images of Fig. $2 \mathrm{a}-\mathrm{d}$. In (a), in close proximity $(7.4 \mu \mathrm{m})$ to the apex, the mean terrace width is $458 \mathrm{~nm}$. In this image only large terraces are present, consisting of a topological ridge with decreasing steps to either side. The step edges meander considerably and their overall alignment deviates significantly from the expected direction along $(11 \overline{2})$ (vertical direction in the image). The STM image in Fig. 2b, $19 \mu \mathrm{m}$ from the apex, shows significant step bunching in combination with large terraces containing vacancy islands. The largest terrace spans up to $1200 \mathrm{~nm}$, whereas the mean TW in this image is $211 \mathrm{~nm}$. While still meandering considerably, the steps are more aligned along the direction determined by the crystal curvature. Vacancy islands are only observed on wider terraces. In Fig. 2c at $135 \mu \mathrm{m}$ from the apex, step bunching is reduced and we observe a clear reduction in the distribution of terrace widths. Step edges now follow the expected direction but still meander slightly. This difference is likely due (at least in part) to the disappearance of vacancy islands relatively close to a step edge being "absorbed" by the edge during the final annealing cycle. Further from the apex large-scale meandering is no longer observed and vacancy islands are absent. Figure $2 \mathrm{~d}$ shows parallel and equidistant step edges in the expected direction with an average TW of $4.24 \mathrm{~nm}$ and a maximum terrace width of $5.23 \mathrm{~nm}$.

For a perfect surface as created by the truncated bulk, we expect the only 'defect sites' to be the highly kinked step edges sketched in Fig. 1c appearing in our STM images as lines running perpendicular to the curvature of the crystal. As shown in Fig. 2, near the apex the number of defect sites is increased due to meandering steps and vacancy islands. By quantifying the lengths of all steps, including those forming vacancy islands in our STM images, we can obtain the ratio between intentional and unintentional defect sites. We divide by the expected TW at that position to create a normalized value. The value equals 1 in case the defect density equals that of an array of steps with an average terrace width as expected at that position for the ideally truncated bulk. In case more defects remain, the value increases. The results in Fig. 2e plot the ratio of total defect sites to expected defect sites, $\frac{d_{\text {tot }}}{d_{\text {exp }}}$ as a function of distance from the apex (lower axis) and step density (upper axis). Blue data indicates that the image(s) used to determine defect lengths contained vacancy islands. Red data reflect results for images that did not show these islands. Within the first $30 \mu \mathrm{m}$ on either side of the apex, we find a significant surplus in defect sites. This is also where we observe the most vacancy islands. Between 30 and $100 \mu \mathrm{m}$, as the surface approaches what we observe in Fig. 2d, the defect ratio approaches 1 . Beyond $100 \mu \mathrm{m}$ from the apex, the presented data is estimated based on terrace widths alone as vacancy islands do not occur. The value fluctuates around 1. These STM-based results and those shown for the LEED analysis in the supporting information, allow us to conclude that the applied cleaning procedure for this crystal yields the expected defect density along the crystal's curvature from a step density $\leq 0.005 \mathrm{~nm}^{-1}$, i.e. an expected $\mathrm{TW} \leq 200 \mathrm{~nm}$ ). For wider terraces, significant numbers of defects, both in the form of vacancy islands and bunched steps, occur. These increase the expected surface defect concentration with a factor of $\sim 10^{1}$ very close to the apex.

\subsection{Determining the Chirality of Kinked Surface Arrays on the Curved Crystal}

Figure $2 \mathrm{f}$ exemplifies one of the vacancy islands found on the surface close to the apex. In all STM images we only find vacancy islands that are a single atomic layer deep, indicating that during our last sputter step at elevated temperature, the surface is strictly in a layer-by-layer removal regime. The subsequent, final annealing step at $773 \mathrm{~K}$, is carried out at a temperature where vacancy islands on $\mathrm{Pt}(111)$ can assume their thermally equilibrated shape [49]. Clearly, 
(a)

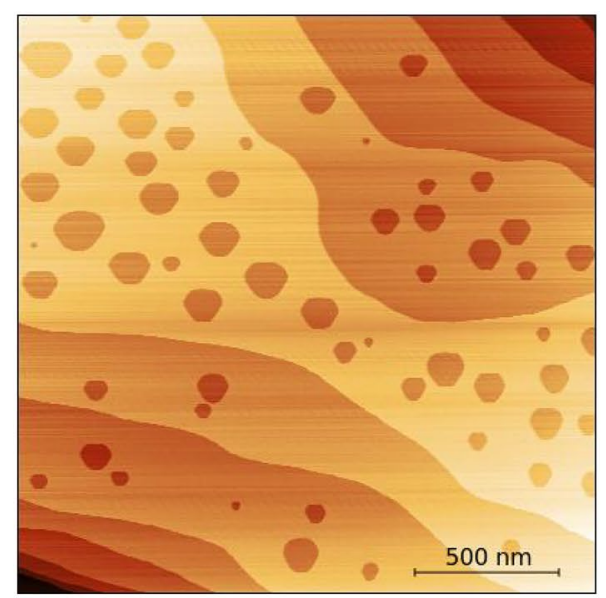

(c)

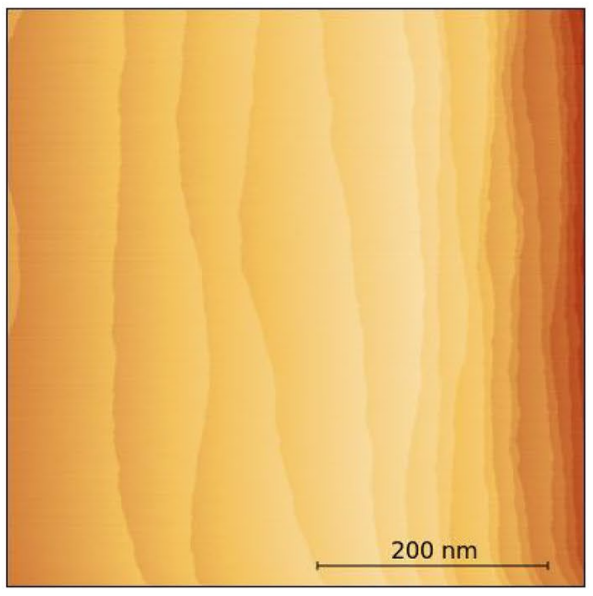

(e)

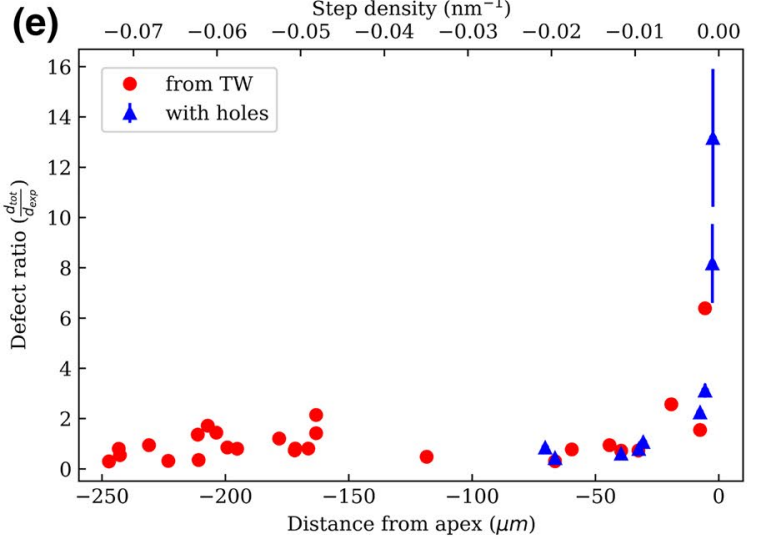

Fig. 2 Typical STM images obtained close to the (111) apex. a distance to apex: $7.4 \mu \mathrm{m}$; mean TW: $211 \mathrm{~nm}$, $\max$ TW: $1200 \mathrm{~nm}, \mathbf{b}$ distance to apex: $-19 \mu \mathrm{m}$; mean TW: $148 \mathrm{~nm}$, max TW: $357 \mathrm{~nm}$, c distance to apex: $-135 \mu \mathrm{m}$; mean TW: $8.7 \mathrm{~nm}$, $\max$ TW: $24 \mathrm{~nm}$, d mean TW: $-800 \mu \mathrm{m}$; mean TW: $8.4 \mathrm{~nm}$, $\max$ TW: $22 \mathrm{~nm}$. e Defect ratio of line defects and vacancy islands as a function of distance from the apex (with expected step density indicated on top axis). Red (b)

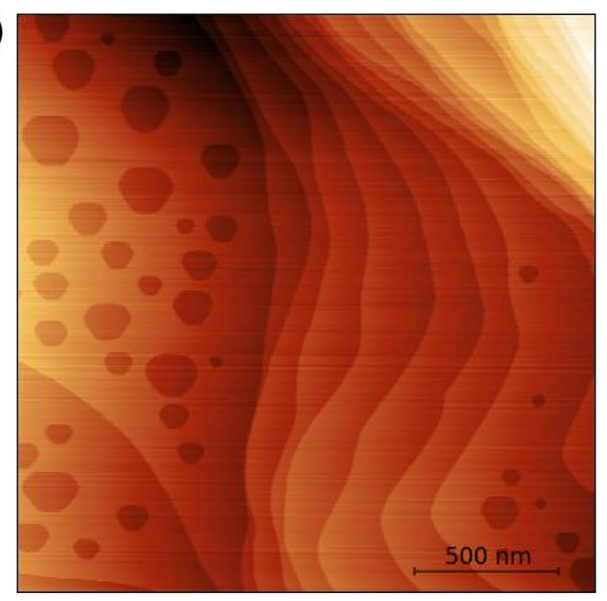

(d)

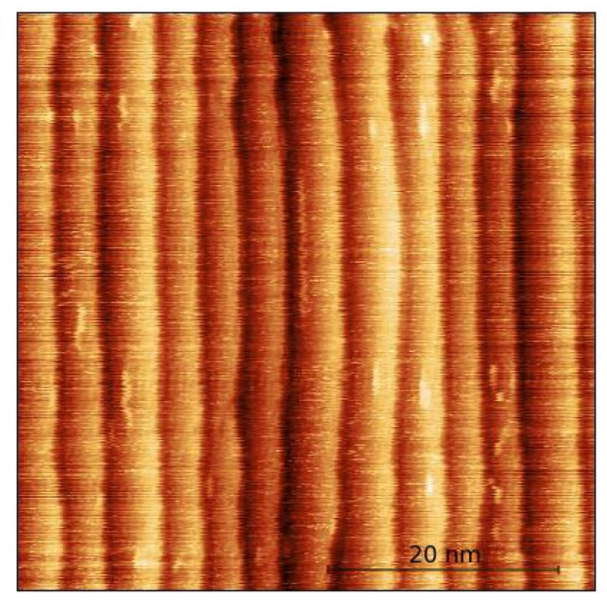

(f)

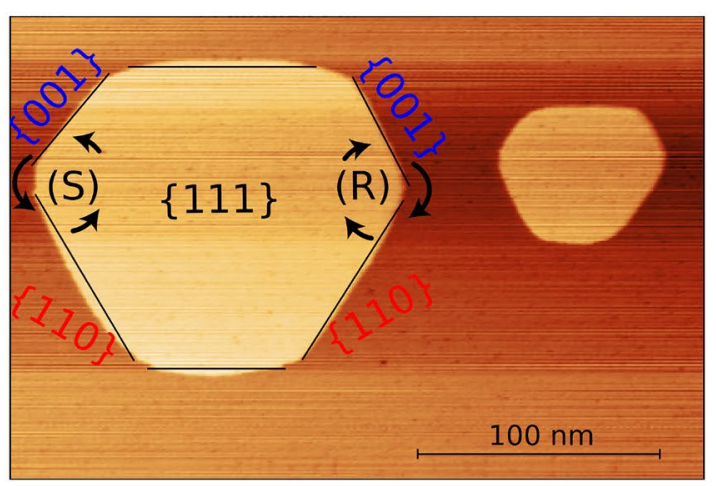

circles represent surfaces with no vacancy islands. Blue triangles represent images with vacancy islands. f Vacancy islands with slightly deformed hexagonal shape. The relative length of $\{001\}$ and $\{110\}$ microfacets (A- and B-type steps) is determined by their line free energies. Step types are indicated in the image and allow the determination of chirality at inner corners and subsequently allow us to determine the chirality of stepped surfaces on either side of the crystal 


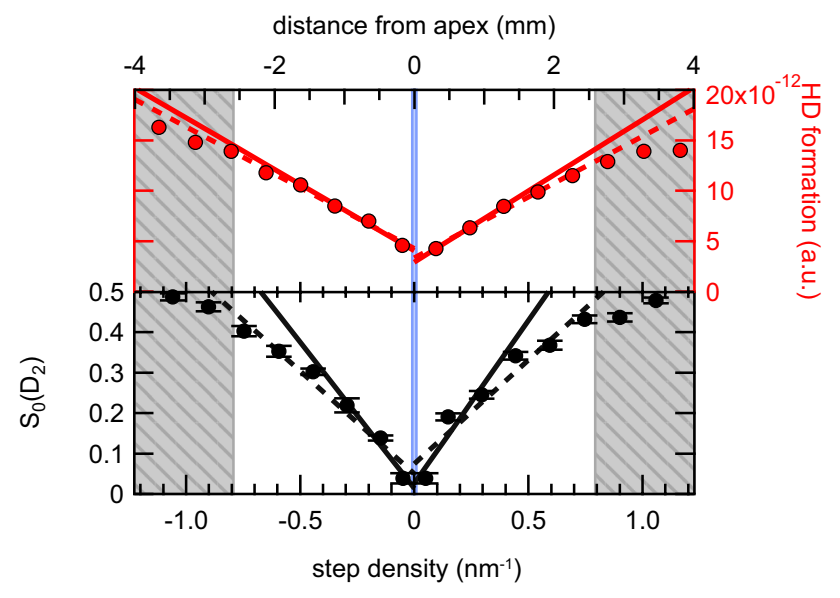

Fig. $3 \mathrm{D}_{2}$ sticking probability, $\mathrm{S}_{0}$ (black circles) and HD formation (red circles) across the curved crystal. Expected step densities (bottom axis) are calculated as a function of distance from the apex (top axis). The blue shaded region in the center highlights the region of the crystal where the defect ratio on the crystal is above 1 , and thus the observed step density exceeds the expected step density. The lowest two circles, measured at the apex, are shifted to the actual step density probed by the molecular beam at the apex. Grey shaded areas indicate where the molecular beam becomes wider than the single crystal's surface; data in the grey areas are not included in any fits. Solid lines are fits to the data including points up to $1 \mathrm{~mm}$ from the center. Dashed lines are fits including data up to $2.6 \mathrm{~mm}$ from the center, i.e. all data in the white area of the plot. Fit lines are extrapolated to a step density of zero, as well as to step densities at the outermost parts of our crystal

the annealing time was not sufficient for all vacancies to be removed. The equilibrium shape can be determined as the Wulff construction of $\{001\}$ and $\{110\}$ facets. The length ratio between short and long edges (at equilibrium) then correlates to the line free energy of A- and B-type steps, as shown by Michely et al. [49] We have analysed 20 different vacancy islands at 10 locations close to the crystal's apex. The average ratio of long-to-short edge was determined as $0.65 \pm 0.06$. This agrees very well with the value obtained by Michely et. al at a similar annealing temperature. Since $\{110\} / B-t y p e$ steps are energetically favoured [50], we can identify the longer step edges as B-type steps and shorter edges as A-type steps.

All of the many vacancy islands appearing in our STM images have the same relative orientation. In combination with the identification of step types forming these islands, we can determine the chirality of stepped surfaces on either side of the crystal. First, we identify the chirality of the corners inside the vacancy islands. We then realize that the left side of a vacancy island reflects the steps on the right side of the curved crystal and vice versa. In Fig. 2f, the short edges are marked, in blue, with $\{001\}$ and the long edge with $\{110\}$, in red. We notice that the right corner of the vacancy island, which steps down towards the left, has a clockwise rotational chirality, denoted $(R)$. Vice versa, in the left corner we observe a counter-clockwise chirality denoted by $(S)$, stepping down towards the right of the crystal. The inherent vacancy defects near the (111) apex of our crystal thus enables us to determine the chirality of the crystal's sides, opening the possibility for controlled chiral adsorption and reactivity experiments.

\section{3 $\mathrm{D}_{2}$ Dissociation and HD Formation on the Curved Crystal}

The detailed insights into the structure of the kinked surfaces along our curved crystal provide a background to revisit previously determined dissociation probabilities for $\mathrm{D}_{2}$ on this this crystal [34]. $S_{0}\left(\mathrm{D}_{2}\right)$ has been shown to be nearly temperature independent between 100 and $350 \mathrm{~K}$ for $\mathrm{Pt}(111)$ and vicinal surfaces.[51-55] We therefore expect no appreciable differences between the surface structures found in the STM study at room temperature, and during the molecular beam study at $155 \mathrm{~K}$. Figure 3 shows in the bottom half of the graph the absolute dissociation probabilities of $\mathrm{D}_{2}$ versus step density. The grey shaded areas indicate the part of the curved crystal that has a reduced width due to tapering of the crystal's edges. The molecular beam is wider here than the crystal. Our measurements in this range, therefore, do not necessarily reflect the actual dissociation probability accurately. We omit the data in these regimes from our analysis and arguments.

The dissociation probabilities in Fig. 3 increase approximately linearly from the (111) surface towards higher step densities at the sides of the crystal. We find no significant difference between the left and right sides of the crystal. This is expected for $\mathrm{D}_{2}$ dissociation, as there is no reason to assume enantiomeric selectivity. The STM analysis presented in Fig. 2e shows that when terraces are narrower than $200 \mathrm{~nm}$, the ratio of total defects over intended defects hovers around 1, i.e. unintended contributions to the defect density are absent. Furthermore, STM images in this range predominantly show rather well-defined arrays of terraces. We may thus safely conclude that indeed the increasing reactivity results from the linear increase in step density.

In Fig. 2e, we found that the defect density did not reflect the expected value very close to the apex. The blue shaded area in the plot marks this range of the curved crystal's surface. The observed number of defects is considerably higher than the expected step density. The size of this area is clearly very small in comparison to the range over which the apparent linear dependence of the dissociation probability appears. This area may be even smaller during molecular beam experiments, when the last annealing step was carried out at a slightly higher temperature than before STM imaging. In fact, it is already twice smaller than the area covered by rectangularly-shaped molecular beam. Consequently, although the defect density may be one order of magnitude 
higher than the expected value in the blue shaded area, the absolute defect density that the beam probes at the center is on average still very small, and reactivity in the area close to the apex is expected to mostly be due to dissociation at the terraces. The quality of a fit that includes the data at or near the apex is not influenced much by the increased defect density.

The slope extracted from a linear fit to the data represents the reactivity of the step in a somewhat odd unit of nm. It represents the average length measured normal to the edge over which the step influences the impinging molecule such that it leads to dissociation [32]. Multiplied by the unit cell width, we obtain the absolute differential reaction cross-section in $\mathrm{nm}^{2}$ for the kinked step at the determined collision energy [34]. To improve upon the value determined earlier, we now include the dissociation probability measured at the apex, but displace it parallel to the $\mathrm{x}$-axis to the position that reflects the same defect density as determined by STM imaging. Also, we note that, although the data seem linear with defect density over the regime that we may include in our analysis, some curvature appears beyond $\sim 1.5 \mathrm{~mm}$ from the apex. Hence, we successively apply a linear fit function to increasing data from the apex. A fit that only includes the data up to $1 \mathrm{~mm}$ from the apex represents those data very well with a slope of $0.764 \mathrm{~nm}$ (averaged over both sides). This fit overestimates reactivity at positions further away than $2 \mathrm{~mm}$ from the apex. Hence the fit adequately covers the reactivity dependence on step density from 'infinite' to $\sim 6$ atom wide terraces. Including additional data up to 2.6 $\mathrm{mm}$ from the apex, corresponding to an average of 4.5 atom wide terraces, gives a slope of $0.510 \mathrm{~nm}$.

The cause for the non-linearity is not clear at this time. We consider that our measurements may miss a small amount in the measured reactivity for narrower terraces as the local surface area is quite reactive. Judging from the original data, we do not believe this to be true though. Also, some physical process may lower the chance for a molecule to dissociate when terraces are very narrow. It is well-known that the grating formed by the step arrays for several transition metals becomes better defined, i.e. shows reduced terrace width distributions, with decreasing terrace width $[56,57]$. We wonder whether improved molecular diffraction from these highly ordered areas near the edge causes increased scattering at the expense of dissociation.

Reversing the direction of extrapolation, we consider our fits at the center of the plot, i.e. at a step density of absolute zero. We thus obtain the reactivity for a "perfect", i.e defect free (111) surface. Being able to do so is a great advantage when comparing measured reactivities to those obtained in theoretical studies. Such comparisons usually have to accept that while experimental studies will always be carried out on a surface having a residual defect density, theoretical studies are usually computed on perfectly constructed (111) surfaces and cannot easily capture the effect of statistical defects. For the initial sticking probability of $\mathrm{D}_{2}$ we reach slightly different values for zero step density, namely $0.026 \pm 0.06$ and $0.014 \pm 0.03$ for the fits involving the data up to $1 \mathrm{~mm}$ from the apex. For the fits over the wider range, up to 2.6 $\mathrm{mm}$ from the apex, we find $0.075 \pm 0.03$ and $0.050 \pm 0.02$. While there is some disparity between those values, they indicate the range in which to expect sticking probabilities for a perfect (111) surface. The initial sticking probability $S_{0}$ experimentally obtained closest to the apex, $0.039 \pm 0.013$, falls within that range.

The upper half of Fig. 3 shows HD formation resulting from a mixed $\mathrm{H}_{2} / \mathrm{D}_{2}$ molecular beam. These data again show a linear increase with step density. Both sides of the apex also yield nearly identical results. Here, we carry out linear fits once more, first for the data within $1 \mathrm{~mm}$ of the apex, then for the range up to $2.6 \mathrm{~mm}$ from the apex. We note that the data does not show the same curvature as the $S_{0}\left(\mathrm{D}_{2}\right)$ data. The fits show a similar slope and offset, regardless of the included fitting range.

For HD to be produced, the following elementary reactions are relevant:

$$
\begin{array}{r}
\mathrm{H}_{2}+2 * \rightleftharpoons 2 \mathrm{H}_{\mathrm{ads}} \\
\mathrm{D}_{2}+2 * \rightleftharpoons 2 \mathrm{D}_{\mathrm{ads}} \\
\mathrm{H}_{\mathrm{ads}}+\mathrm{D}_{\mathrm{ads}} \rightarrow \mathrm{HD}
\end{array}
$$

The two equations expressing the dissociation of $\mathrm{H}_{2}$ and $\mathrm{D}_{2}$ are equilibria as the surface bound $\mathrm{H}_{\text {ads }}$ and $\mathrm{D}_{\text {ads }}$ atoms produced by dissociation of $\mathrm{H}_{2}$ and $\mathrm{D}_{2}$ may desorb with their original partner or another atom of the same isotope. Only if they encounter and desorb with an atom of the other isotopic type, is HD formed, as expressed in the third reaction. For the dissociation equilibria, the probability for dissociation equals $S_{0}$.

To understand the relative kinetics of HD formation, several aspects are important. First, for the kinetic energy used here, there is also no significant temperature dependence to reactivity. This is important as we will compare the dependence in dissociation measured at $300 \mathrm{~K}$ to the $\mathrm{H}-\mathrm{D}$ exchange at $500 \mathrm{~K}$. Second, none of the supersonic molecular beam studies of hydrogen dissociation on $\mathrm{Pt}(111)$ and stepped Pt surfaces has ever reported an isotope effect, while all surfaces show a slightly different kinetic energy dependence [51-55, 58]. As we use a single beam containing both $\mathrm{H}_{2}$ and $\mathrm{D}_{2}$, their velocities are (nearly) identical. Their values for $S_{0}$ at the conditions used here, therefore, differ. For a single surface type, e.g. $\operatorname{Pt}(111)$, we can estimate the instantaneously produced surface concentrations of $\mathrm{H}_{\text {ads }}$ and $\mathrm{D}_{\mathrm{ads}}$ from $\mathrm{S}_{0}$ and the flux. However, the measured reactivity along the curved surface may be considered a combination of the reactivity on (111) planes and steps. The previously reported kinetic energy dependencies for $\mathrm{Pt}(111)$ and highly 
stepped surfaces are inverted, but vary with the surface. On $\mathrm{Pt}(111)$, reactivity only increases with kinetic energy, while on all stepped surfaces the reactivity initially drops with kinetic energy prior to it increasing again. This makes it difficult to unravel the ratio of produced $\mathrm{H}_{\mathrm{ads}}$ and $\mathrm{D}_{\mathrm{ads}}$ from the impinging molecules. We make no attempt to estimate them here. However, it is unlikely that the expected statistical 1:2:1 ratio of $\mathrm{H}_{2}: \mathrm{HD}: \mathrm{D}_{2}$ product formation is achieved in our experiments as a result of unequal concentrations of $\mathrm{H}_{\mathrm{ads}}$ and $\mathrm{D}_{\mathrm{ads}}$.

On the other hand, we note that the rate of HD formation increases only by a factor of 3.4 over the entire range from the apex to the edge where our molecular beam fits onto the crystal's surface, i.e. at 4-5 atom wide terraces. The dissociation probability measured in the same range increases by at least a factor of 7.5, depending on the exact value used for $\mathrm{S}_{0}$ at $\operatorname{Pt}(111)$. HD production, therefore, does not track the change in elementary dissociation. We interpret this observation as possibly suggesting that the higher binding energy of $\mathrm{H}(\mathrm{D})$ atoms at steps limits their mobility and diffusion, hence increasing the chance to desorb with their original partner. Shifting the balance in the two equilibria of the homonuclear isotopologues puts formation of the heteronuclear isotopologues at a disadvantage, reducing the rate of HD formation relative to the dissociation. The impact of diffusion on the rate of product formation was recently also implied in a study of CO oxidation on Pt(111) and stepped surfaces [22]. There also, steps break the symmetry of the (111) plane and cause diffusion to be non-isotropic.

\section{Outlook and Conclusion}

We have presented a structural analysis of a curved Pt single crystal with (111) at the apex and kinked surfaces towards the sides. We find that, with the applied cleaning procedure, most parts of the surface are cleaned thoroughly and form ordered stepped arrays. Using the equilibrated shape of residual vacancy islands near the apex, we can identify Aand B-type steps within the kinked step edges and determine the chirality of stepped surfaces on either side of the apex. This opens possibilities for chemical experiments probing chiral activity. The unintentional defects in close proximity to the apex are abundant, but do not significantly affect experiments that probe chemical reactivity towards achiral reactions, i.e. $\mathrm{D}_{2}$ dissociation and HD formation. For both reactions we find a (nearly) linear increase with step density. For the dissociation, it reconfirms that steps provide much higher dissociation probabilities than terrace sites and that, over a wide range, the measured reactivity may be taken as a linear sum of individual contributions by steps and terraces. A slight deviation from linearity at the highest step densities may be indicative of improved diffractive scattering from highly ordered step arrays or another mechanism that lowers reactivity. The linearity of the entire range, but considerably lower dependence on step density for HD production, may indicate anisotropic diffusion caused by higher binding of $\mathrm{H}$ (D) atoms at steps sites. Lastly, we note the potential of curved crystals to extract reactivities for perfect, defectfree flat surfaces, such as (111), forging an important bridge between experimental and theoretical results.

Author Contributions All authors contributed to the study conception and design. Material preparation, data collection and analysis were performed by TR and SA. They contributed equally to the manuscript. All authors read and approved the final manuscript.

Funding Leiden Institute of Chemistry, Leiden University.

\section{Compliance with Ethical Standards}

Conflict of interest The authors declare that they have no conflict of interest.

Open Access This article is licensed under a Creative Commons Attribution 4.0 International License, which permits use, sharing, adaptation, distribution and reproduction in any medium or format, as long as you give appropriate credit to the original author(s) and the source, provide a link to the Creative Commons licence, and indicate if changes were made. The images or other third party material in this article are included in the article's Creative Commons licence, unless indicated otherwise in a credit line to the material. If material is not included in the article's Creative Commons licence and your intended use is not permitted by statutory regulation or exceeds the permitted use, you will need to obtain permission directly from the copyright holder. To view a copy of this licence, visit http://creativecommons.org/licenses/by/4.0/.

\section{References}

1. Muiño RD, Busnengo HF (eds) (2013) Dynamics of gas-surface interactions. Series in surface science. Springer, New York

2. Buurmans IL, Weckhuysen BM (2012) Heterogeneities of individual catalyst particles in space and time as monitored by spectroscopy. Nat Chem 4(11):873-886

3. Honkala K, Hellman A, Remediakis IN, Logadottir A, Carlsson A, Dahl S et al (2005) Ammonia synthesis from first-principles calculations. Science 307(5709):555-558

4. Al-Abadleh HA, Grassian VH (2003) Oxide surfaces as environmental interfaces. Surf Sci Rep 52(3-4):63-161

5. Bell AT (2003) The impact of nanoscience on heterogeneous catalysis. Science 299(5613):1688-1691

6. Liang D, Abend G, Block J, Kruse N (1983) Formation of nickel subcarbonyls from nickel and carbon monoxide. Surf Sci 126(1-3):392-396

7. Block J, Kruse N (1987) Chemical surface reactions of CO on the atomic scale: investigations by field ion microscopy and mass spectrometry. React Kinet Catal Lett 35(1-2):11-20

8. Voss C, Kruse N (1996) Oscillatory behavior in the catalytic reduction of $\mathrm{NO}$ and $\mathrm{NO} 2$ with hydrogen on Pt field emitter tips. Appl Surf Sci 94:186-193 
9. Voss C, Kruse N (1998) Chemical wave propagation and rate oscillations during the $\mathrm{NO} 2 / \mathrm{H} 2$ reaction over Pt. Ultramicroscopy 73(1-4):211-216

10. Visart de Bocarmé T, Kruse N (2002) Kinetic instabilities during the NOx reduction with hydrogen on Pt crystals studied with field emission on the nanoscale. Chaos 12(1):118-130

11. de Bocarmé TV, Chau TD, Kruse N (2006) Imaging and probing catalytic surface reactions on the nanoscale: field ion microscopy and atom-probe studies of O2-H 2/Rh and NO-H 2/Pt. Top Catal 39(1-2):111-120

12. Barroo C, De Decker Y, Visart de Bocarmeé T, Kruse N (2014) Complex oscillation patterns during the catalytic hydrogenation of NO2 over platinum nanosized crystals. J Phys Chem C 118(13):6839-6846

13. Barroo C, De Decker Y, Bocarmeé T, Gaspard P (2015) Fluctuating dynamics of nanoscale chemical oscillations: theory and experiments. J Phys Chem Lett 6(12):2189-2193

14. Barroo C, Voorsluijs V, de Bocarmé TV, Gaspard P, De Decker Y (2018) Reconstructing stochastic attractors from nanoscale experiments on a non-equilibrium reaction. Phys Chem Chem Phys 20(33):21302-21312

15. McEwen JS, Gaspard P, De Decker Y, Barroo C, Visart de Bocarmeé T, Kruse N (2010) Catalytic reduction of NO2 with hydrogen on Pt field emitter tips: kinetic instabilities on the nanoscale. Langmuir 26(21):16381-16391

16. Kleyn A (2003) Molecular beams and chemical dynamics at surfaces. Chem Soc Rev 32(2):87-95

17. McCormack DA, Olsen RA, Baerends EJ (2005) Mechanisms of $\mathrm{H} 2$ dissociative adsorption on the $\mathrm{Pt}(211)$ stepped surface. J Chem Phys 122(19):194708

18. den Dunnen A, Wiegman S, Jacobse L, Juurlink LBF (2015) Reaction dynamics of initial O2 sticking on Pd(100). J Chem Phys 142(21):214708. https://doi.org/10.1063/1.4921571

19. Gutiérrez-González A, Crim FF, Beck RD (2018) Bond selective dissociation of methane $(\mathrm{CH} 3 \mathrm{D})$ on the steps and terraces of Pt (211). J Chem Phys 149(7):074701

20. Smeets EWF, Füchsel G, Kroes GJ (2019) Quantum Dynamics of Dissociative Chemisorption of $\mathrm{H} 2$ on the Stepped $\mathrm{Cu}(211)$ Surface. J Phys Chem C 123(37):23049-23063

21. Chadwick H, Gutiérrez-González A, Beck RD, Kroes GJ (2019) CHD3 dissociation on the kinked $\mathrm{Pt}(210)$ surface: a comparison of experiment and theory. J Phys Chem C 123(23):14530-14539

22. Neugebohren J, Borodin D, Hahn HW, Altschäffel J, Kandratsenka A, Auerbach DJ et al (2018) Velocity-resolved kinetics of site-specific carbon monoxide oxidation on platinum surfaces. Nature 558(7709):280-283

23. Zhou L, Kandratsenka A, Campbell CT, Wodtke AM, Guo $\mathrm{H}$ (2019) Origin of thermal and hyperthermal CO2 from CO oxidation on Pt Surfaces: the role of post-transition-state dynamics, active sites, and chemisorbed CO2. Angew Chem 131(21):6990-6994

24. Cao K, van Lent R, Kleyn AW, Kurahashi M, Juurlink LBF (2019) Steps on Pt stereodynamically filter sticking of O2. Proc Natl Acad Sci USA 116(28):13862-13866

25. Gutiérrez-González A, Torio M, Busnengo H, Beck RD (2019) Site selective detection of methane dissociation on stepped $\mathrm{Pt}$ surfaces. Top Catal 62(12-16):859-873

26. Chadwick H, Gutiérrez-González A, Migliorini D, Beck RD, Kroes GJ (2018) Incident angle dependence of CHD3 dissociation on the stepped $\mathrm{Pt}(211)$ surface. J Phys Chem C 122(34):19652-19660

27. Chadwick H, Gutiérrez-González A, Beck RD, Kroes GJ (2019) Transferability of the SRP32-vdW specific reaction parameter functional to CHD 3 dissociation on $\mathrm{Pt}(110)-(2 \times 1)$. J Chem Phys 150(12):124702. https://doi.org/10.1063/1.5081005
28. Muttaqien F, Hamamoto Y, Inagaki K, Morikawa Y (2014) Dissociative adsorption of $\mathrm{CO} 2$ on flat, stepped, and kinked $\mathrm{Cu}$ surfaces. J Chem Phys 141(3):034702

29. Poelsema B, Lenz K, Comsa G (2010) The dissociative adsorption of hydrogen on defect- 'free'Pt (111). J Phys 22(30):304006

30. Poelsema B, Lenz K, Comsa G (2011) The dissociative adsorption of hydrogen on Pt (111): actuation and acceleration by atomic defects. J Chem Phys 134(7):074703

31. Auras SV, Juurlink LBF. Recent advances in the use of curved single crystal surfaces. Progress in Surface Science to be published;

32. Van Lent R, Auras SV, Cao K, Walsh AJ, Gleeson MA, Juurlink LBF (2019) Site-specific reactivity of molecules with surface defects-the case of $\mathrm{H} 2$ dissociation on Pt. Science 363(6423):155-157

33. Schiller F, Ilyn M, Peérez-Dieste V, Escudero C, Huck-Iriart C, Ruiz del AN et al (2018) Catalytic oxidation of carbon monoxide on a curved Pd crystal: spatial variation of active and poisoning phases in stationary conditions. J Am Chem Soc 140(47):16245-16252

34. Auras SV, van Lent R, Bashlakov D, Piñeiros Bastidas JM, Roorda $\mathrm{T}$, Spierenburg R, et al (2020) Scaling Pt-catalyzed hydrogen dissociation on corrugated surfaces. Angewandte Chemie international Edition

35. Hahn E, Schief H, Marsico V, Fricke A, Kern K (1994) Orientational instability of vicinal Pt surfaces close to (111). Phys Rev Lett 72(21):3378

36. Comsa G, Mechtersheimer G, Poelsema B (1980) He beam scattering and LEED evidence for partial facetting of a Pt (997) surface. Surf Sci 97(1):L297-L303

37. Sander M, Imbihl R, Ertl G (1992) Kinetic oscillations in catalytic CO oxidation on a cylindrical Pt single crystal surface. J Chem Phys 97(7):5193-5204

38. Schweicher J, Bundhoo A, Frennet A, Kruse N (2014) Dynamic hydrogen-deuterium exchange to determine metallic surface areas of catalysts. Catal Lett 144(2):204-210

39. Mcfadden CF, Cremer PS, Gellman AJ (1996) Adsorption of chiral alcohols on "chiral" metal surfaces. Langmuir 12(10):2483-2487

40. Michely T, Land T, Littmark U, Comsa G (1992) Morphological effects induced by the formation of a Pt-adatom lattice gas on $\mathrm{Pt}$ (111). Surf Sci 272(1-3):204-210

41. King DA, Wells MG (1972) Molecular beam investigation of adsorption kinetics on bulk metal targets: Nitrogen on tungsten. Surf Sci 29(2):454-482

42. Lu K, Rye R (1974) Flash desorption and equilibration of H2 and D2 on single crystal surfaces of platinum. Surf Sci 45(2):677-695

43. Christmann K, Ertl G (1976) Interaction of hydrogen with Pt (111): the role of atomic steps. Surf Sci 60(2):365-384

44. van der Niet MJ, den Dunnen A, Juurlink LB, Koper MT (2010) The influence of step geometry on the desorption characteristics of O 2, D 2, and $\mathrm{H} 2 \mathrm{O}$ from stepped Pt surfaces. J Chem Phys 132(17): 174705

45. Kolb MJ, Garden AL, Badan C, Torres JAG, Skúlason E, Juurlink LB et al (2019) Elucidation of temperature-programmed desorption of high-coverage hydrogen on Pt (211), Pt (221), Pt (533) and $\mathrm{Pt}$ (553) based on density functional theory calculations. Phys Chem Chem Phys 21(31):17142-17151

46. Badan C, Koper MT, Juurlink L (2015) How well does Pt (211) represent Pt [n (111) $\times(100)]$ surfaces in adsorption/desorption? J Phys Chem C 119(24):13551-13560

47. van Lent R (2019) Steps in gas-surface reactions. PhD thesis, Leiden University

48. Cao K (2018) Structure dependence of molecular reactions on surfaces. PhD thesis, Leiden University

49. Michely T, Comsa G (1991) Temperature dependence of the sputtering morphology of $\mathrm{Pt}(111)$ 
50. Lahee AM, Manson JR, Toennies JP, Wöll C (1986) Observation of interference oscillations in helium scattering from single surface defects. Phys Rev Lett 57(4):471-474

51. Luntz A, Brown J, Williams M (1990) Molecular beam studies of $\mathrm{H} 2$ and D2 dissociative chemisorption on Pt (111). J Chem Phys 93(7):5240-5246

52. Gee A, Hayden B, Mormiche C, Nunney T (2000) The role of steps in the dynamics of hydrogen dissociation on $\mathrm{Pt}(533)$. $\mathrm{J}$ Chem Phys 112(17):7660-7668

53. Groot IM, Kleyn AW, Juurlink LB (2011) The energy dependence of the ratio of step and terrace reactivity for $\mathrm{H} 2$ dissociation on stepped platinum. Angew Chem Int Ed 50(22):5174-5177

54. Groot I, Schouten K, Kleyn A, Juurlink L (2008) Dynamics of hydrogen dissociation on stepped platinum. J Chem Phys 129(22):224707

55. Cao K, van Lent R, Kleyn A, Juurlink L (2018) A molecular beam study of D2 dissociation on Pt (1 111 ): Testing SRP-DFT calculations. Chem Phys Lett 706:680-683
56. Walter AL, Schiller F, Corso M, Merte LR, Bertram F, LoboCheca J et al (2015) X-ray photoemission analysis of clean and carbon monoxide-chemisorbed platinum (111) stepped surfaces using a curved crystal. Nat Commun 6(1):1-7

57. Ortega JE, Corso M, Abd-el Fattah Z, Goiri E, Schiller F (2011) Interplay between structure and electronic states in step arrays explored with curved surfaces. Phys Rev B 83(8):085411

58. Samson P, Nesbitt A, Koel B, Hodgson A (1998) Deuterium dissociation on ordered Sn/Pt (111) surface alloys. J Chem Phys 109(8):3255-3264

Publisher's Note Springer Nature remains neutral with regard to jurisdictional claims in published maps and institutional affiliations. 